\title{
Management of incidental pancreatic cysts: which guidelines?
}

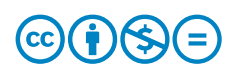

\author{
Author \\ Kevin McGrath \\ Institutions \\ Division of Gastroenterology, Hepatology and Nutrition, University \\ of Pittsburgh Medical Center, Pittsburgh, Pennsylvania, United \\ States \\ submitted 3.11 .2016 \\ accepted after revision 2.1.2017
}

\author{
Bibliography \\ DOI http://dx.doi.org/10.1055/s-0043-102399 | \\ Endoscopy International Open 2017; 05: E209-E211 \\ (c) Georg Thieme Verlag KG Stuttgart · New York \\ ISSN 2364-3722
}

\section{Corresponding author}

Kevin McGrath, MD, Division of Gastroenterology, Hepatology and Nutrition, University of Pittsburgh Medical Center, 200 Lothrop St M2, C Wing, Pittsburgh, PA 15213 P

Phone: +1-412-648-9325

Fax: +1-412-383-8992

mcgrathkm@upmc.edu
Medicine is not a perfect science. That is no truer than in the evaluation and management of the asymptomatic pancreatic cyst. Ever more common, these cystic lesions frustrate gastroenterologists, surgeons and patients alike. Existing consensus statements and societal guidelines have been based upon expert opinion and little evidence, frequently leaving physicians who care for these patients with more questions than answers. The recent American Gastroenterological Association (AGA) guidelines, which many view as controversial, have continued to stir this pot.

From a historical perspective, pancreatic cyst guidelines have continued to evolve over the last decade. The first guidelines (American Society for Gastrointestinal Endoscopy, 2005) recommended endoscopic ultrasound-guided fine-needle aspiration (EUS-FNA) of all cysts for cyst fluid analysis and cytology [1]. The Sendai guidelines (International Association of Pancreatology, 2006) specifically addressed Intraductal papillary mucinous neoplasms and mucinous cystic neoplasms, and called for more selective use of EUS based on cyst size and presence of worrisome features [2]. The updated Fukuoka guidelines (International Association of Pancreatology, 2012) similarly recommended the presence of a worrisome feature for evaluation by EUS; cyst size became less of a concern. Worrisome features include cyst size size $\geq 30 \mathrm{~mm}$, enhanced thickened cyst walls, non-enhanced mural nodules, main pancreatic duct (MPD) size 5 to $9 \mathrm{~mm}$, abrupt change in MPD caliber with distal glandular atrophy, and lymphadenopathy. Criteria for surgical referral according to the Fukuoka guidelines are any high-risk stigmata which include obstructive jaundice in the setting of a pancreatic head cyst, presence of a mural nodule/solid component, MPD $\geq 10 \mathrm{~mm}$ or cytology suspicious or positive for malignancy [3]. The current AGA guidelines (2015) deal with management of asymptomatic cysts, without regard to cyst type. They recommend the presence of 2 high-risk stigmata (cyst size size $\geq 30 \mathrm{~mm}$, dilated MPD, solid component) before an
EUS examination be performed. For surgical referral, the AGA guidelines require malignant cytology on EUS-FNA or at least 2 high-risk features (cyst size size $\geq 30 \mathrm{~mm}$, dilated MPD, solid component)[4]. Thus, with time and knowledge, guidelines have become more conservative with the recognition that the malignant risk of an asymptomatic cyst is very low. Accordingly, magnetic resonance imaging (MRI) for surveillance is increasingly recommended.

In this edition of Endoscopy International Open, 2 articles pour more gas on the fire. Lee et al.'s retrospective 2-part study evaluates and compares the operating characteristics of the AGA and Fukuoka guidelines for detection of and surgical referral for malignant pancreatic cysts [5]. An EUS database was used to determine appropriateness for EUS referral of a MRI-detected asymptomatic cyst $(n=143)$ based on criteria for such according to the 2 sets of guidelines. For EUS detection of a high-risk cyst (HGD or carcinoma), the Fukuoka guidelines were more sensitive (33.3\% vs $16.7 \%$ ) but less specific $(65.4 \%$ vs $94.4 \%$ ) compared to the AGA guidelines. This is no surprise given the guidelines' criteria for EUS referral. Twenty three cysts were referred for surgery based on EUS findings; 7 were malignant or harbored HGD. Using definitive cytology and/or surgical histology, based on AGA guidelines, 5 of the 7 (71.4\%) high-risk cysts did not meet criteria for EUS referral, where 3 of 7 (42.9\%) did not meet criteria by the Fukuoka guidelines.

The second part of the study analyzed the guidelines for referral to surgical resection utilizing a pathology database of resected asymptomatic cysts $(n=152)$ that had undergone MRI and EUS. Based on criteria for surgical referral, the performance characteristics did not differ statistically between the guidelines; $30.9 \%$ and $36.2 \%$ of patients would have been referred by the AGA and Fukuoka guidelines, respectively. For high-risk cysts based on surgical pathology $(n=17), 5$ of $17(29.4 \%)$ did not meet surgical criteria by AGA guidelines; 3 of these 17 (17.6\%) did not meet criteria by Fukuoka guidelines. 
According to both sets of guidelines, $75 \%$ of the surgical resections in this study were considered unnecessary. Certainly, if AGA guidelines are followed, there will be a decrease in EUS procedures and surgical resections (at the risk of missing advanced neoplasia). However, it is somewhat unfair to apply these current guidelines to a surgical practice that spanned from 1995 to 2013, which was the time period for the surgical portion of the study. Given our knowledge deficits at the time, I suspect many cyst resections from 1995 to 2005 would have been considered unnecessary by today's standards, regardless of either guideline.

The Ge et al. study retrospectively applied the AGA guidelines to a large multicenter cohort of pancreatic cysts $(n=300)$ which underwent surgical resection from 2004-2014 [6]. All patients had undergone preoperative EUS. The AGA guidelines would have recommended surgery in $40 \%$ of these patients; of them, $37 \%$ where ultimately found to have advanced neoplasia, defined by the presence of cancer or HGD. Sixty percent of the cohort would have avoided surgery, with cancer being missed in $5 \%$.

On the surface, it appears that the AGA guidelines may have performed fairly well in the Ge et al. study, correctly recommending surveillance in $95 \%$ of patients. However, if we further dissect the results, 54 total patients in the study were found to have malignant pancreatic cysts. Nine of 54 patients had missed cancers; these had no evidence of concerning cytology and fewer than 2 high-risk features. Of the remaining 45 patients with malignant cysts, 24 patients were recommended for surgery solely on the basis of concerning cytology. Fifteen of these 24 patients had only one high-risk feature, and 9 had no high-risk features. Therefore, if AGA guidelines for cyst management were strictly applied, these 24 patients would have never been referred for EUS (where FNA resulted in the finding of concerning cytology). Thus, by following AGA guidelines, 33/ $54(61 \%)$ of cancers would have been missed. Granted, this was a biased study, as an inclusion criterion was EUS examination.

Clearly, these guidelines are still deficient in their ability to identify and appropriately refer high-risk pancreatic cysts for EUS and/or surgery. By increasing the threshold to "qualify" for EUS assessment and surgical referral, we will miss more cysts with curable disease (high grade dysplasia and early carcinoma). According to the Lee et al. study, $71 \%$ of high-risk cysts did not meet criteria for EUS referral and $29 \%$ did not meet surgical referral criteria, per the AGA guidelines. Sixty-one percent of cancers (by my calculation) would not have been appropriately referred in the Ge et al. study. Is this acceptable?

Our goal should be to identify the patients with HGD and early carcinoma as they are the ones to most benefit from surgical intervention (which obviously has to be individualized and risk-stratified). By increasing the thresholds, the AGA guidelines have decreased sensitivity but increased specificity. Detecting advanced cancer will offer little to the patient.

In my opinion, there is little argument that cyst fluid analysis (carcinoembryonic antigen, viscosity, molecular analysis, cytology) via EUS-FNA is helpful to differentiate mucinous from nonmucinous cysts. The Ge et al. study fortifies the role of EUS and cytology in cyst management. And it is possible that the role of
EUS will only grow with technologic advances in cyst fluid and cyst wall evaluation. We have evolving tools to image and sample the wall (confocal laser endomicroscopy, forceps biopsy), along with increasing capability to detect DNA mutations to predict biologic behavior. The AGA guidelines manage all cysts similarly without defining whether they are mucinous or not, which is problematic. I believe it is crucial to differentiate mucinous from non-mucinous lesions early on, as the non-mucinous (mostly serous cystadenomas) do not require surveillance. This may actually be cost-saving in the long run.

The time is now for multicenter prospective evaluation of guidelines and new algorithms. It is not fair to apply current guidelines to a time when we knew far less than we do nowhence no surprise that current guidelines would have retrospectively "prevented" surgeries. Our clinical decision-making has evolved with experience and natural history data.

So which guideline should one follow? For now, I believe common sense should prevail in the context of patient individualization. Strict adherence to current guidelines is appropriate for approved prospective validation studies. In my opinion, the most pressing issue is the cyst size threshold for EUS assessment, as few will argue that the presence of any other worrisome or high risk feature warrants EUS. Our group recommended $1.5 \mathrm{~cm}$ [7]. Lee et al. and the Fukuoka guidelines recommend $2 \mathrm{~cm}$ as a size threshold $[3,5]$ and the AGA recommends $3 \mathrm{~cm}[4]$. Which shall it be?

Medicine is still not a perfect science. But it's time for the evaluation of pancreatic cystic lesions to become a more precise science, to allow for the evolution of real evidence-based guidelines.

\section{Competing interests}

None

References

[1] Jacobson BC, Baron TH, Adler DG et al. ASGE guideline: the role of endoscopy in the diagnosis and management of cystic lesions and inflammatory fluid collections of the pancreas. Gastrointest Endosc 2005; 61: $363-370$

[2] Tanaka M, Chari S, Adsay V et al. International consensus guidelines for management of intraductal papillary mucinous neoplasms and mucinous cystic neoplasms of the pancreas. Pancreatology 2006; 6: $17-32$

[3] Tanaka M, Fernandez-del Castillo C, Adsay V et al. International consensus guidelines 2012 for the management of IPMN and MCN of the pancreas. Pancreatology 2012; 12: $183-197$

[4] Vege SS, Ziring B, Jain R et al. American Gastroenterological Association institute guideline on the diagnosis and management of asymptomatic neoplastic pancreatic cysts. Gastroenterology 2015; 148: $819-822$

[5] Lee A, Kadiyala V, Lee L. Evaluation of AGA and Fukuoka guidelines for EUS and surgical resection of incidental pancreatic cysts. Endosc Int Open 2017; 05: E116-E122 
[6] Ge PS, Muthusamy VR, Gaddam S et al. Evaluation of the 2015 AGA guidelines on pancreatic cystic neoplasms in a large surgically confirmed multicenter cohort. Endoscop Int Open 2017; 05: E201 -E208

[7] Singhi AD, Zeh H], Brand RE et al. American Gastroenterological Association guidelines are inaccurate in detecting pancreatic cysts with advanced neoplasia: a clinicopathological study of 225 patients with supporting molecular data. Gastrointest Endosc 2016; 83: 1107 1117 